\title{
Beyond Blue: a Novel In-flight Dinner Ritual
}

\author{
Fleur A. van Midwoud \\ Delft University of Technology
}

Beyond Blue is a food tray design for serving meals in intercontinental flights. Whereas conventional food trays are flat and cover most of the passenger's tabletop, Beyond Blue is a foldable box that, when opened, changes into a stable tray of similar size as the conventional models (Figure 1, right). When closed, the box covers less than a third of the tray table (Figure 1, left). Beyond Blue's main added value is that it enables passengers to create a personalized meal ritual with a self-controlled beginning (opening the box) and ending (reclosing the box).

Figure 2 visualizes the Beyond Blue usage scenario. When the meal is served, the passenger can either open the box immediately or decide to wait for some time, for example to finalize other activities (e.g. finishing a book page or having a bathroom visit). The passenger has the ability to end their meal at any point in time with the simple action of closing the box. Once closed, the empty food containers are inside the box and out of sight, creating a tidy appearance, and taking up only little space of the tray table. Moreover, the closed box is a subtle sign to the cabin crew that the passenger has finished his or her meal.

\section{Design challenge}

This design was part of the author's Master's thesis in Integrated Product Design at the Delft University of Technology, The Netherlands (Van Midwoud, 2014). The project was executed in collaboration with the In-flight Services department of KLM Royal Dutch Airlines. The target group of the project was Economy Class passengers on non-stop long-haul flights, which are long-distance journeys typically last eight hours or more. Because KLM had identified (with customer satisfaction assessments) that passengers generally experience long-haul flights as energy-drainers, the main aim of the current project was to design "something" that would enable the passengers to feel more energized when arriving at their destination. The topic of design was not predefined but to be determined in the project.

Fleur A. van Midwoud, Industrial Design Engineering, Delft University of Technology.

Correspondence concerning this article should be addressed to Fleur A. van Midwoud, Delft University of Technology, Faculty of Industrial Design Engineering, Landbergstraat 15, 2628 CE Delft, The Netherlands E-mail:fleur.vanmidwoud@gmail.com

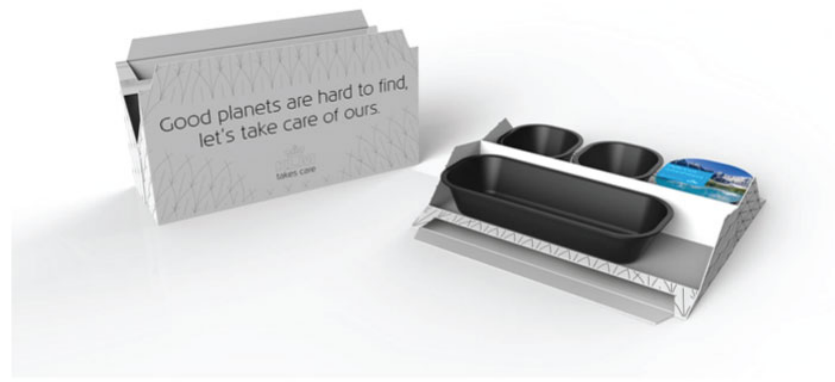

Figure 1. Beyond Blue in-flight meal serving tray.

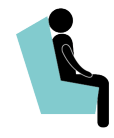

After sitting in his seat in the same environment for a long time, the passenger starts feeling bored.

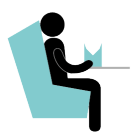

After finishing the meal, the passenger stacks the left overs and fold the tray, the option to take own initiative is appreciated.

Figure 2. Beyond Blue usage scenario.

The change of shape makes it for the crew easy to see who finished their meal and who did not yet.

The meal is handed to the passenger, he immediatly has a clear overview of the provided products.

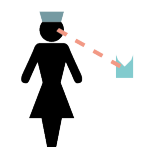

The first step was to identify the energy-drainers; in-flight moments that cost positive energy. In terms of reversal theory, an important aim was to understand these energydrainers in relation to the passengers' motivational states. This relation is important because reversal theory proposes that there is not a one-to-one relationship between arousal and positive energy (Apter, 2007). High arousal represents positive energy when someone is in a paratelic state. However, when the same person is in a telic state, arousal stimulates negative experiences like anxiety, representing negative energy. Hence, understanding in-flight energy-drainers, 
required a focus on the interplay between the passengers' arousal and motivational state.

\section{Project approach}

The project was structured as an iterative process with several qualitative research and design steps (see Van Midwoud, 2014). In this process, design prototypes were generated to test and to further explore initial insights. An explorative questionnaire was administered during an 11-hour flight to Mexico City. The questionnaire covered the relation between arousal and motivational states by including questions about motivation, psychodiversity, and energy. In addition, a creative session was organized with cabin-crew, using the carousel of feelings tool (see Van Midwoud et al., this issue) to tap into the flight attendants' expertise and experiences. These explorations resulted in five initial design directions. Three directions were selected and prototypes were developed that were tested by regular passengers in a long-haul flight. The additional insights drawn from this test served as inspiration for the Beyond Blue design concept.

\section{Insights and design implications}

The several research executions indicated that, once settled in, passengers generally stay in the paratelic motivational state, driven by momentary experiences rather than by specific goals. In this state, low arousal is experienced as boredom and dullness (Apter, 2007). This explains the observation that (when not sleeping) most passengers tend to avoid low arousal by keeping themselves busy with, for example, the entertainment system or a book. In line with this, it was expected that, as a distraction and source of pleasure, meal servings would be an additional source of positive arousal. A key finding, however, was that although the meal servings generated the expected increase in arousal, they also stimulated a reversal from the paratelic to the telic state shortly after the meal was finished. While the meal servings provided the passenger with challenges (i.e. how and when to eat the meal and what to do with the food containers), the required "confidence frame" was not sufficiently in place for the passengers to be confident about their abilities to deal with these challenges. As a consequence, the increased arousal was experienced as negative rather than positive. The confidence frame was not in place because, in the conventional situation, the eating experience is controlled by the cabin crew: they decide when the meal is served and cleared away. Moreover, once served, the tray fills up the complete table, dominating the passengers' activities. After eating the meal, the (half) empty food containers, dirty cutlery and napkins remain in front of the passenger. The passengers do not have the means and the space to clean this up, leaving them with no option but to sit in front of their trash until the crew has time to clear the tables. As a result, the passenger enters into a kind of survival mode during the meal servings, in which they experience unpleasant arousal due to the experienced lack of control. The passenger tries to regain control by engaging in goal-oriented behavior, like eating everything in the short amount of time available, or trying to make a stack of the trash after eating the meal. Since high arousal is not enjoyed in the telic state, the following design opportunity was identified: redesign the meal servings with the aim to minimize the reversal from the paratelic to the telic state in order to stimulate enjoyable arousal, enabling the passengers to enjoy the moment and not worry about what happens later. This resulted in Beyond Blue, which was designed with the aim to provide passengers with some control over their eating experience with the simple ritual of opening and closing the box that provides a modest but essential level of control.

\section{Discussion}

In this project, reversal theory enabled the designer to understand observed user experiences in relation to underlying motivational states. Traditionally, the designer would look for direct (causal) relationships between user experience and design variables. Reversal theory provided the option to follow the users' experiences back to the source by reasoning how the motivation evolves. This added valuable meaning to the research findings because it facilitated a holistic understanding of the passengers' experience in relation to their motivation and behavior. One aspect of reversal theory that proved to be challenging is that it is difficult to predict which motivational state users are in. But the theory suggests which state someone would be in to as result of a change in context. Designers are used to working with situations in which they know the starting point, while reversal theory requires one to refocus from the starting point to the desired situation. A second challenge is the inability to precisely predict in which motivational state a person will be in a certain situation. The type of situation and the person's activity can give clues to the motivational state she is in (e.g. the paratelic state while watching a movie), but the more unpredictable factors of satiation and frustration can also have pushed the person is into a different state. This is a challenge for designers who want to predict motivational states to make a suitable design. Nevertheless, reversal theory proved to be valuable as an integrating theory that enabled the designer to integrate rich and diverse research insights into an integrated and constructive design direction.

\section{References}

Apter, M. J. (2007). Reversal Theory: The Dynamics of Motivation, Emotion and Personality (2nd. ed). Oxford: Oneworld Publications.

Van Midwoud, F.A. (2013). Beyond Blue: a user focused food tray design to enhance the KLM in-flight experience. Unpublished Master's thesis. Delft (NL): Delft University of Technology. 